\title{
EDITORIAL
}

\section{Report from London}

Prostate Cancer and Prostatic Diseases (2010) 13, 103;

doi:10.1038/pcan.2010.10

It is with more than a tinge of sadness that I sit down to compose my very last editorial for Prostate Cancer and Prostatic Diseases. In June this year myself and Judd Moul are relinquishing the editorship of this Journal, which we have seen flourish since its inception in 1997, with a rising impact factor and ever more submissions from prestigious units around the world.

Over the past 12 years many profound changes have occurred in the diagnosis and management of prostatic diseases, nearly all of which have been reported in, or commented upon, in the pages of this journal. The molecular genetics of prostatic cancer are being gradually teased out, with implications for targeted screening. New molecular diagnostic tests, such as PCA3 and TMPRESS-2, based on the overexpression of specific genes, can now help to distinguish prostate cancer from $\mathrm{BPH}$. New ways of removing the cancerous prostate, laparoscopically and with robotic assistance, have reduced the collateral damage from surgery and speeded patients' recovery. During that time other minimally invasive therapies, including high-intensity focused ultrasound and cryotherapy, have been gaining ground. The introduction of taxane-based chemotherapy has prolonged the survival of those faced with hormonerelapsed metastatic prostate cancer and newer treatment options for this condition are in the pipeline.

BPH management has similarly metamorphosed. Combination therapy with an $\alpha$-blocker and a 5- $\alpha$ reductase inhibitor has been shown, both in the MTOPS trial and the CombAT study, to be more effective than either class of compound alone. Holmium laser enucleation of the benignly enlarged prostate is now seriously challenging TURP. Unfortunately, similar progress has not been seen in the Cinderella subject of prostatitis, which remains, to paraphrase Winston Churchill, an enigma wrapped in a mystery. More research clearly needs to be carried out in this disease area.
In this issue of Prostate Cancer and Prostatic Diseases, as always, many of these key areas are addressed. For example, we start with a very pertinent review of the optimization of docetaxel in men with castration-resistant metastatic prostate cancer by Armstrong and George. This is followed by an original report pertaining to the identification of chromosomal translocation, $\mathrm{t}(4 ; 6)(\mathrm{q} 22 ; \mathrm{q} 15)$, in prostate cancer from Shan et al. The issue of accurate prediction of repeat prostate biopsy outcomes by a mitochondrial DNA deletion assay is addressed by Robinson et al.

The effectiveness and morbidity of transrectal highintensity focused ultrasound treatment of localized prostate cancer is reviewed by Ripert et al. While the time course of late rectal toxicity after radiation therapy for prostate cancer is the subject of a paper by Odrazka et al. A paper from Kyrdalen et al. examines fatigue in hormone-naive prostate cancer patients treated with radical prostatectomy or definitive radiotherapy. Integrin involvement in freeze resistance of androgen-insensitive prostate cancer is examined by Baust et al. All of these treatments cost money, substantial amounts of it, so the retrospective analysis illustrating the substantial clinical and economic burden of prostate cancer by E David Crawford et al. is especially relevant.

In conclusion, I would like to take the opportunity to thank all of the authors and reviewers for all their hard work and the editorial team, especially my co-editor Judd Moul, as well as Joyce-Rachel John and Nicola Bentham for their dedicated and unswerving support. It has been a privilege to have worked with you all. I would also like to wish the new editor the very bestonwards and upwards we go!

\section{Conflict of interest}

The author declares no conflict of interest.

R Kirby

Co-Editor 